\title{
Comparison of immunological properties of various bioactive combinations
}

\author{
Vaclav Vetvicka, Jana Vetvickova
}

\begin{abstract}
Background. Lately, more and more preparation of various cocktails or mixtures of bioactive modulators have been introduced. Their true activity is, however, rarely tested.

Aim. To compare six commercially available, glucan-based immunostimulators.

Methods. Immunological effects of tested combinations were measured by evaluation of phagocytosis of synthetic particles by peripheral blood neutrophils, production of IL-2 by mouse splenocytes, production of superoxide anion and nitrite oxide, antibody response to imunization with ovalbumin, and NK cell activity.

Results. Our results showed that with the exception of the highest doses (phagocytosis) and superoxide anion and nitrite oxide production, only RVB 300 showed significant immunostimulative activity.

Conclusion. Based on our results, we can conclude that most of the tested natural immunomodulators have limited, if any, biological effects. Only RVB 300 significantly stimulated all six tested immunological reactions.
\end{abstract}

Key words: glucan, phagocytosis, IL-2, NK cells, antibodies

Received: April 20, 2012; Accepted: June 18, 2012; Available online: September 21, 2012

http://dx.doi.org/10.5507/bp.2012.065

Department of Pathology, University of Louisville, Louisville, KY, USA

Corresponding author: Vaclav Vetvicka, e-mail: Vaclav.vetvicka@louisville.edu

\section{INTRODUCTION}

Biologically active, cheap, safe and natural modulators of the immune system have been sought throughout history. Some, like $\beta$-glucan, were intensively studied and now, 14,000 plus publications later, are being tested in numerous clinical trials ${ }^{1}$. However, despite clear and wellestablished biological effects of this immunomodulator, the search for even better effects continues. Lately, more and more manufacturers are experimenting with the preparation of various cocktails or mixtures of potentially bioactive powders. It is now very common to find glucan in combination with five or more ingredients, including Echinacea, Aloe vera, Astragalus, and Goldenseal.

The major problem with these combinations is that there is very little research that supports any beneficial effects. Individual components usually are biologically active, but the effects of the combinations are rarely tested. This is particularly true in the case of complex extracts such as the extracts of Echinacea ${ }^{2,3}$. There are literally hundreds of different parts and we have absolutely no clue as to their biological activities. Some substances will have no activity, some might stimulate, and some might inhibit the immune system. However, there are studies showing that some bioactive molecules have synergistic effects when combined with glucan. Numerous scientific studies have shown beneficial effects when glucan was given in combination with vitamin $\mathrm{C}^{4}$. Additional studies have shown the positive influence of dietary glucan supplemented with vitamin $\mathrm{C}$ on both non-specific and specific immune responses of carp and rainbow trout ${ }^{5}$.

Our own studies demonstrated significant stimulation of both cellular (phagocytosis, tumor suppression) and humoral (antibody production and cytokine secretion) branches of immune reaction with a combination of glucan and humic acid ${ }^{6}$. Another study showed that both glucan and resveratrol stimulated the phagocytosis of blood leukocytes, caused the increased expression of CD4 on spleen cells, and showed higher restoration of spleen recovery after experimentally induced leucopenia. In all cases, strong synergetic effects were observed. When we measured the effects of these substances on the expression level of some important genes (such as NF- $k$ B2, $\mathrm{Cdc} 42$ and Bcl-2) in breast cancer cells, the up-regulation of Cdc42 expression was evident only with the use of both immunomodulators in combination ${ }^{7,8}$. Follow-up experiments showed that compared to the individual components, the glucan-resveratrol-vitamin $\mathrm{C}$ combination was the strongest activator of phagocytosis and antibody response and the strongest inhibitor of experimentallyinduced lung and breast cancer 9 .

Natural immunomodulators are slowly becoming a mainstream supplement and, with dozens of clinical trials under way, their use in regular clinical practice is only a question of time. Based on limited published comparison of individual natural immunomodulators ${ }^{10,11}$ and no comparison of various combinations, we decided to compare numerous commercially available combinations of immunomodulators.

\section{MATERIAL AND METHODS}

\section{Animals}

Female, 8 week old BALB/c mice were purchased from the Jackson Laboratory (Bar Harbor, ME). All animal work was done according to the University of 
Louisville IACUC protocol. Animals were sacrificed by $\mathrm{CO}_{2}$ asphyxiation.

\section{Material}

Individual samples were purchased from the manufacturers or distributors as shown in Table 1. RPMI 1640 medium, sodium citrate, Wright stain, Limulus lysate test E-TOXATE, Concanavalin A, HEPES, PMA, cytochrom C, LPS, penicillin and streptomycin were obtained from Sigma Chemical Co. (St. Louis, MO). Fetal calf serum (FCS) was from Hyclone Laboratories (Logan, UT).

\section{Cell lines}

Human neutrophil cell line HL-60, human myeloid cell line U937 and YAC cells were obtained from the ATCC (Manassas, VA). The cell lines were maintained in RPMI 1640 medium containing HEPES buffer supplemented with $10 \%$ heat-inactivated FCS, $100 \mathrm{U} / \mathrm{mL}$ penicillin and $100 \mu \mathrm{g} / \mathrm{mL}$ streptomycin, in plastic disposable tissue culture flasks at $37{ }^{\circ} \mathrm{C}$ in a $5 \% \mathrm{CO}_{2} / 95 \%$ air incubator.

\section{Phagocytosis}

The technique employing phagocytosis of synthetic polymeric microspheres was described earlier ${ }^{12,13}$. Briefly: peripheral blood cells were incubated in vitro with 0.05 $\mathrm{mL}$ of 2-hydroxyethyl methacrylate particles (HEMA; $\left.5 \times 10^{8} / \mathrm{mL}\right)$. The test tubes were incubated at $37{ }^{\circ} \mathrm{C}$ for $60 \mathrm{~min}$, with intermittent shaking. Smears were stained with Wright stain. The cells with three or more HEMA particles were considered positive. Mice were injected ip. with individual samples or PBS (control). All experiments were performed in triplicate. At least 200 cells in 60 high power fields were examined in each experiment.

\section{IL-2 production}

Purified spleen cells $\left(2 \times 10^{6} / \mathrm{mL}\right.$ in RPMI 1640 medium with $5 \%$ FCS) from mice injected with tested samples were added into wells of a 24-well tissue culture plate. After addition of $1 \mathrm{mg}$ of Concanavalin A (positive control), cells were incubated for $48 \mathrm{~h}$ in a humidified incubator $\left(37{ }^{\circ} \mathrm{C}, 5 \% \mathrm{CO}_{2}\right)$. At the endpoint of incubation, supernatants were collected, filtered through $0.45 \mathrm{~mm}$ filters and tested for the presence of IL-2 using a Quantikine mouse IL-2 kit (R\&D Systems, Minneapolis, MN).

\section{Antibody formation}

Formation of antibodies was evaluated using ovalbumin as an antigen. Mice were injected twice (two weeks apart) with $0.1 \mathrm{mg}$ of ovalbumin and the serum was collected 7 days after last injection. Experimental groups were getting daily ip. injections of tested material. Level of specific antibodies against ovalbumin was detected by ELISA. As positive control, combination of ovalbumin and Freund's adjuvant was used.

\section{Superoxide and nitrite production}

Cells were incubated in a final volume of $200 \mu \mathrm{l}$ of medium containing $0.1 \%$ gelqatin and $100 \mu \mathrm{M}$ cytochrome C. Mice were challenged with $100 \mathrm{ug}$ of individual glucans
$24 \mathrm{~h}$ earlier. Cell lines were incubated with $1 \mathrm{ug} / \mathrm{mL}$ of glucans for $24 \mathrm{~h}$. For the superoxide production, the reaction was initialized by addition of $5 \mathrm{ng} / \mathrm{ml}$ PMA. After gentle mixing, the absorbance was measured 30 minutes after incubation at $37^{\circ} \mathrm{C}$ using multiwell spectrophotometer at $550 \mathrm{~nm}$. Results are expressed as nanomoles of cytochrome $\mathrm{C}$ reduced/2.5 x $10^{5}$ cells/30 min, after subtraction of the SOD and spontaneous release controls ${ }^{14}$.

For a nitrite $\left(\mathrm{NO}_{2}^{-}\right)$formation we used a technique described by Green and $\mathrm{Nacy}^{15}$ with LPS as triggering agent.

\section{In vitro cytotoxicity assay}

Spleen cells were isolated from spleen of mice by standard methods. Cell suspension was generated by pressing minced spleen against the bottom of a petri dish containing PBS. After elimination of erythrocytes by $10 \mathrm{~s}$ incubation in distilled water, and five washes in cold PBS, the cells were resuspended in PBS and counted. The viability was determined by trypan blue exclusion. Only cells with viability better than $95 \%$ were used in subsequent experiments. Splenocytes $\left(10^{6} / \mathrm{mL} ; 0.1 \mathrm{~mL} /\right.$ well $)$ in V-shaped 96-well microplates were incubated with individual samples $(2 \mu \mathrm{g} / \mathrm{mL})$ for $30 \mathrm{~min}$ at $37{ }^{\circ} \mathrm{C}$ and then washed three times with RPMI 1640 medium. After washing, 50 $\mu \mathrm{l}$ of target cell line YAC-1 (two different concentrations of target cells were used so the final effector-target ratio was $32: 1$, and $64: 1)$. After spinning the plates at $250 \mathrm{x}$ $g$ for $5 \mathrm{~min}$, the plates were incubated for $4 \mathrm{~h}$ at $37^{\circ} \mathrm{C}$. The cytotoxic activity of cells was determined by the use of CytoTox 96 Non-Radioactive Cytotoxicity Assay from Promega (Promega, Madison, WI, USA) according to the manufacturer's instructions. Briefly, $10 \mathrm{ul}$ of lysis solution was added into appropriate control wells $45 \mathrm{~min}$ before the end of incubation. The next step was to spin the plates at $250 \mathrm{x} \mathrm{g}$ for $5 \mathrm{~min}$, followed by transferring 50 ul of supernatant into flat-bottomed, 96-well microplates. After $50 \mathrm{ul}$ of reconstituted substrate was added into each well, plates were covered and incubated for 30 min at room temperature at dark. The optical density was determined by using a STL ELISA reader (Tecan U.S., Research Triangle Park, NC) at 492 nm. Specific cellmediated cytotoxicity was calculated using the formula:

Percent-specific killing (\% cytotoxicity) $=100 \mathrm{x}$ $\left[\left(\mathrm{OD}_{492}\right.\right.$ experimental - $\mathrm{OD}_{492}$ spontaneous $)$ divided $\left(\mathrm{OD}_{492}\right.$ maximum - $\mathrm{OD}_{492}$ spontaneous)] as described in manufacturer's instructions, where spontaneous release was target cells incubated with medium alone and maximum release was that obtained from target cells lysed with the solution provided in the kit.

\section{RESULTS}

The number of various combinations of natural immunomodulators is almost as great as the number of individual compounds. The rationale for their formulation is usually a combination of clever marketing with honest efforts to prepare the immunomodulator with optimal biological effects. For our study, we picked six easily avail- 
able, commercially successful combinations, based (at least partly) on polysaccharides. Information about individual formulations and manufacturers is given in Table 1.

Immunomodulators are generally considered to be either nonspecific stimulators of immunity or stimulators of the cellular branch. Therefore, we started our evaluation by measuring the effects on phagocytosis by peripheral blood cells. We used a well-established model of synthetic microspheres based on 2-hydroxymethacrylate ${ }^{16}$. Another advantage of this experimental design is that it was routinely used in the evaluation of various glucans ${ }^{11,17}$, allowing better comparison. The results summarized in Table 2 can be divided into two parts - first, two combinations, Quivana and BioBran showed no activity at all, and second, three other combinations, Transfer Factor Plus, Manapol, and Immunizen, were active only at the two highest doses, 400 and $800 \mu \mathrm{g} /$ mouse. As the good quality glucans are active at the concentrations around
$50 \mu \mathrm{g}$ (ref. ${ }^{11}$ ), the neutrophils are clearly not a target of these materials. The last combination, RVB 300, showed significant stimulation from the lowest dose with a clear dose dependency.

Phagocytosis is closely connected with additional steps used by professional phagocytes to eliminate invading targets. Internalization of prey is often accompanied with an oxidative burst including production of several oxygen species. In this study, we measured the production of superoxide anion and nitrite oxide using two separate models - mouse neutrophils and neutrophil cell line HL60. Data are summarized in Table 3 and show that using fresh cells, only Transfer Factor Plus and RVB 300 stimulated production of superoxide anion, but RVB 300 was $4.5 \mathrm{x}$ stronger. When we used the cell ine HL-60, most of the samples (with exception of Quivana) showed stimulative effects, but again, RVB 300 showed 4.5x stronger effects. Evaluation of nitrite oxide showed similar results (Table 4). In both tests, all tested samples significantly

Table 1. Type of combination used in this study.

\begin{tabular}{|c|c|c|}
\hline Sample & Composition & Source \\
\hline Qivana & $\begin{array}{l}\text { Reishi (Ganoderma lucidum) } \\
\text { Cordyceps (Cordyceps sinensis) } \\
\text { Coriolus (Coriolus versicolor) } \\
\text { Maitake (Grifola frondosus) } \\
\text { Zhu Ling (Polyporus umbellatus) }\end{array}$ & Qivana, USA \\
\hline BioBran & MGN-3 Arabinoxylan Compound & Daiwa Pharmaceutical, Japan \\
\hline Transfer Factor Plus & $\begin{array}{l}\text { Zinc (as zinc methionine) } \\
\text { Transfer Factor E-XF'T } \\
\text { Nano Factor }{ }^{\mathrm{TM}} \\
\text { IP-6 } \\
\beta \text {-Sitosterol and other phytosterols } \\
\text { Cordyceps sinensis mycelia extract } \\
\text { Baker's yeast extract } \\
\text { Agricus blazeii fruiting body extract } \\
\text { Aloe leaf gel extract } \\
\text { Oat seed extract } \\
\text { Olive leaf extract } \\
\text { Maitake (Grifola frondosa) extract } \\
\text { Shiitake (Lentinus edodes) extract }\end{array}$ & 4Life, USA \\
\hline Manapol & $\begin{array}{l}\text { Manapol powder } \\
\text { Beta-1,3-glucan }\end{array}$ & Carrington Laboratories, USA \\
\hline Immunizen & $\begin{array}{l}\text { Colostrum } \\
\text { Arabinogalactan } \\
\text { Beta } 1,3 \text { glucan } \\
\text { Lactoferrin }\end{array}$ & Unicity, USA \\
\hline RVB 300 & $\begin{array}{l}\text { Glucan \#300 (Saccharomyces cerevisiae) } \\
\text { Resveratrol } \\
\text { Vitamin C }\end{array}$ & RYL, USA \\
\hline
\end{tabular}


Table 2. Effects of various combinations on phagocytosis.

\begin{tabular}{lcccccc}
\hline Dose $(\mu \mathrm{g})$ & 25 & 50 & 100 & 200 & 400 & 800 \\
\hline Qivana & $29.1 \pm 1.1$ & $30.3 \pm 1.3$ & $30.5 \pm 1.4$ & $33.7 \pm 1.1$ & $30.1 \pm 2.0$ & $35.1 \pm 1.7$ \\
BioBran & $31.3 \pm 1.3$ & $32.3 \pm 2.1$ & $32.8 \pm 3.3$ & $33.1 \pm 2.1$ & $32.0 \pm 1.8$ & $32.2 \pm 1.5$ \\
TF Plus & $31.2 \pm 2.8$ & $32.6 \pm 0.8$ & $32.0 \pm 1.4$ & $34.3 \pm 1.9$ & $35.0 \pm 2.5$ & $36.8 \pm 2.8^{*}$ \\
Manapol & $30.5 \pm 1.4$ & $31.5 \pm 1.9$ & $36.6 \pm 3.6$ & $38.9 \pm 4.9$ & $40.2 \pm 2.6^{*}$ & $43.8 \pm 3.3^{*}$ \\
Immunizen & $30.8 \pm 0.7$ & $32.9 \pm 1.1$ & $34.0 \pm 2.3$ & $33.5 \pm 2.2$ & $38.9 \pm 2.5^{*}$ & $37.1 \pm 2.1^{*}$ \\
RVB 300 & $40.1 \pm 1.4^{*}$ & $46.9 \pm 2.1^{*}$ & $67.2 \pm 2.9^{*}$ & $73.8 \pm 3.5^{*}$ & $74.5 \pm 4.2^{*}$ & $75.6 \pm 3.7^{*}$ \\
\hline
\end{tabular}

PBS $-31.2 \pm 1.0$

*Significant difference from PBS control at $P<0.05$ level.

Table 3. Effects of various combinations on superoxide anion production.

\begin{tabular}{lcc}
\hline \multirow{2}{*}{ SAMPLE } & \multicolumn{2}{c}{ superoxide anion } \\
& \multicolumn{2}{c}{ (nanomoles per 2.5 x 105 cells) } \\
\cline { 2 - 3 } & Mouse neutrophils & HL-60 \\
\hline Qivana & $0.21 \pm 0.04$ & $0.22 \pm 0.05$ \\
BioBran & $0.37 \pm 0.13$ & $0.34 \pm 0.06^{*}$ \\
TP Plus & $0.52 \pm 0.09 *$ & $0.33 \pm 0.03^{*}$ \\
Manapol & $0.44 \pm 0.13$ & $0.40 \pm 0.17^{*}$ \\
Immunizen & $0.42 \pm 0.12$ & $0.27 \pm 0.04^{*}$ \\
RVB 300 & $1.99 \pm 0.29 * *$ & $1.87 \pm 0.15^{* *}$ \\
PBS & $0.23 \pm 0.07$ & $0.11 \pm 0.02$ \\
\hline
\end{tabular}

*Significant stimulation of superoxide anion production at $P<0.05$ level. **Significant stimulation of superoxide anion production at $P<0.01$ level.

Table 4. Effects of various combinations on nitrite oxide production.

\begin{tabular}{lcc}
\hline \multirow{2}{*}{ SAMPLE } & \multicolumn{2}{c}{$\begin{array}{c}\text { Nitrite oxide } \\
(\mu \mathrm{mol} / \mathrm{L})\end{array}$} \\
& Mouse neutrophils & HL-60 \\
\hline Qivana & $1.34 \pm 0.25^{*}$ & $1.14 \pm 0.26^{*}$ \\
BioBran & $2.43 \pm 0.33^{*}$ & $2.13 \pm 0.27^{*}$ \\
TP Plus & $2.45 \pm 0.38^{*}$ & $2.02 \pm 0.31^{*}$ \\
Manapol & $2.41 \pm 0.26^{*}$ & $2.02 \pm 0.22^{*}$ \\
Immunizen & $1.71 \pm 0.35^{*}$ & $1.14 \pm 0.12^{*}$ \\
RVB 300 & $10.75 \pm 0.29^{*}$ & $7.95 \pm 0.20^{*}$ \\
PBS & $0.24 \pm 0.13$ & $0.11 \pm 0.04$ \\
\hline
\end{tabular}

Stimulation of superoxide anion production was significant at $P<0.01$ level. stimulated nitrite oxide formation, but RVB 300 was always almost five times more active.

Next, we focused on the effects of IL-2 by splenic cells secretion.The production of IL-2 was measured after 48 $\mathrm{h}$ in vitro incubation of cells isolated from control and treated mice. The IL-2 production in spleen cells isolated from control (PBS treated) mice was in range of 0 to 35 pg; the positive control (Concanavalin A) was in (2 500 to $2800 \mathrm{pg}$ ). Our data demonstrated than only Transfer Factor Plus showed very slight stimulation, with RVB 300 reaching $50 \%$ of effects of Concanavalin A (Fig. 1).

The following experiments were focused on the use of tested compounds as an adjuvant. We used an experimental model of immunization of mice with ovalbumin. Mice were injected twice (fourteen days apart) with 0.1 mg of ovalbumin and the serum was collected 7 days after last injection. Experimental groups were getting daily ip. injections of tested material. Freund's adjuvant was used as a positive control. The results (Fig. 2) showed that only RVB 300 significantly increased the antibody response to ovalbumin.

The last part of our study tested the effects of various components on activation of NK cells (Fig. 3). We used a 32:1 ratio and showed that again, only RVB 300 significantly increased the cytotoxicity of NK cells over the PBS control (9.9\%). The increase of effector-target ratio to $64: 1$ showed the same trend (data not shown).

\section{DISCUSSION}

Natural immunomodulators often show remarkable biological and physiological effect, which, together with a current trend towards natural molecules, makes them highly sought after. The most studied biological response modifiers are based on polysaccharides-namely glucans. However, in the never-ending search for even better, safer and more active immunomodulators, various combinations gain popularity.

For this study, we compared six commercial combinations. RVB 300 is recently the subject of several studies ${ }^{9}$ showing strong potential in cancer treatment. Transfer factor from the original studies ${ }^{18}$ was repeatedly tested and evaluated, but interest of the scientific public slowly 


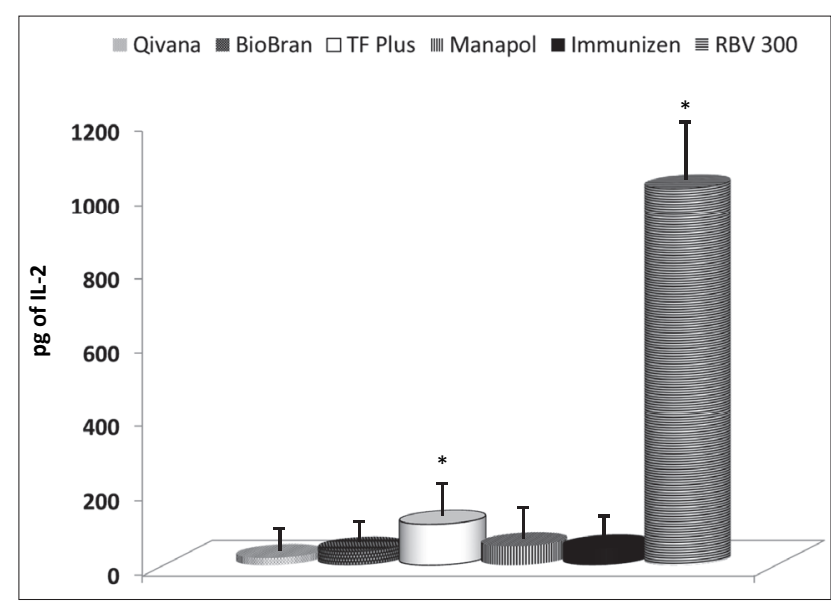

Fig. 1. Effects of tested material on Con A-stimulated secretion of IL-2 by spleen cells. As the control (PBS) production of IL-2 is zero, all collumns represents significant differences between control (PBS) and samples at $P \leq 0.05$ level. All experiments were performed in triplicates.

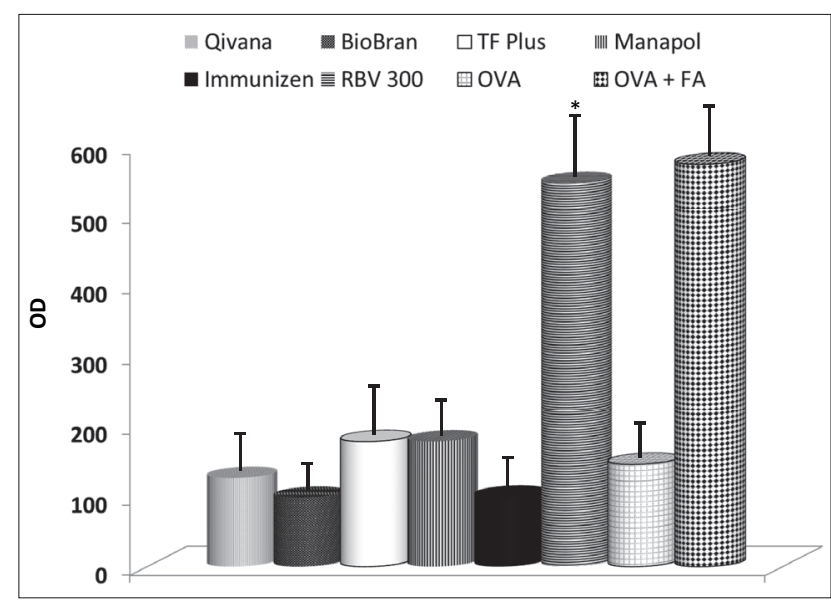

Fig. 2. Effects of two ip. injections of tested samples on formation of antibodies against ovalbumin. Mice were injected twice (two weeks apart) and the serum was collected 7 days after the last injection. The level of specific antibodies against ovalbumin was detected by ELISA. As positive control, Freund's adjuvant was used. *Represents significant differences between the control (ovalbumin alone) and samples at $P \leq 0.05$. Individual substances were used at $100 \mu \mathrm{g} / \mathrm{dose}$. All experiments were performed in triplicate.

diminished despite some promising data ${ }^{19}$. Biobran is a modulator acting mostly on monocytes, T lymphocytes and NK cells ${ }^{20}$ with a synergistic effects with curcumin ${ }^{21}$. The remainder of tested samples is not supported by scientific papers, however the rationale for our choice is their wide availability in all parts of the world.

All polysaccharide-based immunomodulators act primarily on innate immunity and particularly on cellular branch. Therefore, the first reaction tested was phagocytosis with the use of peripheral blood neutrohils and synthetic polymeric particles as a model. While the effects of RVB 300 were consistent with previously published

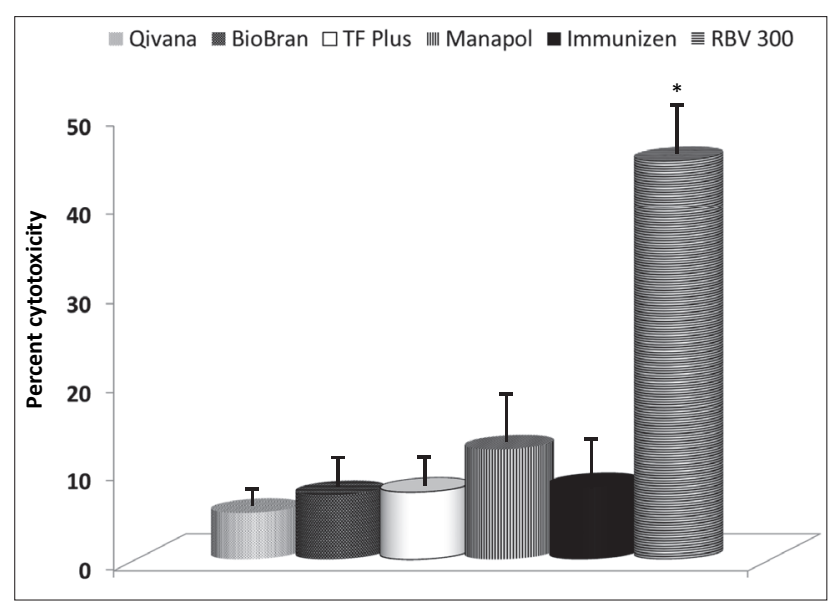

Fig. 3. Effects of tested material on natural killer cell activity against YAC-1 cells. For each experiment, 4 wells per sample were evaluated, each experiment was repeated three times. Control group of mice (6.8\%) was injected with PBS. *Significant at $P<0.005$ level.

results of the resveratrol/glucan/vitamin $\mathrm{C}$ mixture ${ }^{7,9}$, other combinations showed only very limited activity at the highest doses.

Production of active oxygen molecules during oxidative burst is necessary for the destruction of invading microorganisms. Numerous bioactive molecules were found to stimulate oxidative burst ${ }^{22}$. It is, therefore, not surprising that all tested combinations increased both superoxide anion and nitrite oxide production. The highest effects of RVB 300 are most probably caused by combination of glucan and vitamin $\mathrm{C}$, both known for strong stimulation of oxidative burst ${ }^{5,11}$.

Next, we studied the effects of various compounds on NK cell activation. Despite the fact that most combinations claim strong effects on this cell type, the model of YAC-1 killing by activated splenocytes showed only significant effects in one sample.

In addition to direct effects on various cell types, it is assumed that the immunomodulator application results in binding to the receptors with subsequent signalling processing leading to activation of cells and synthesis and release of cytokines and other biologically active molecules. Evaluating the effects on IL-2 production, our study confirmed the strong effects of RVB 300 with limited activity of Transfer Factor Plus. The effects of RVB 300 were almost comparable to the common stimulator Concanavalin A.

Polysaccharides in general and glucans in particular are considered to be stimulators of cellular immunity. However, some recent studies showed that they can not only increase antibody response ${ }^{23}$, but also can serve for robust co-stimulation of cellular and humoral immunity during vaccination ${ }^{24}$. Despite the fact that most of our samples contained glucan (in some case several types of glucan), only RVB 300 showed significant effects.

Data presented in this study clearly demonstrated significant differences among individual types of immunostimulating combinations. With the exception of 
stimulating nitrite oxid and superoxide anion production, five-out-of-six tested samples showed basically no immunological activity, despite presence of otherwise well-documented glucans such as Maitake ${ }^{24}$ or Shiitake glucan. There are two possible explanations - one is that the doses of glucans used in these preparations are not high enough, or that some of the additional parts of these combinations (e.g. colostrum or oat seed extracts) do not work in synergy but in fact negate each other. The first possibility is not readily answered since these compounds state only the total amount of bioactive substances and not their individual doses. The second possibility is difficult to answer as well due to the lack of research evaluating the individual compounds in most of the combinations on the market.

Several conclusions can still be made: 1) most of the commercial immunostimulating combinations have only very limited, if any, effects on the immune system; 2) doses recommended on the labels might not be sufficient; and 3), the resveratrol/glucan/vitamin $\mathrm{C}$ showed the strongest effects. Clearly, more research on possible synergistic effects of individual compounds and on optimal doses of used immunomodulators is necessary to support the commercial claims.

\section{ACKNOWLEGEMENT}

The authors would like to thank Ms. Rosemary Williams for excellent editorial assistance.

\section{CONFLICT OF INTEREST STATEMENT}

Author's conflict of interest disclosure: The authors stated that there are no conflicts of interest regarding the publication of this article.

\section{REFERENCES}

1. Yan J. $\beta$-Glucan-mediated tumor immunotherapy - mechanisms of action and perspective. In: Vetvicka, V., Novak, M. (Eds.), Biology and Chemistry of Beta Glucan - Volume 1: Beta Glucans - Mechanisms of Action, Bentham Science Publishers, 2011, pp. 39-47.

2. Brinkeborn RM, Shah DV, Degenbring FH. Echinaforce and other Echinacea fresh plant preparations in the treatment of the common cold. A randomized, placebo controlled, double-blind clinical trial. Phytomedicine 1999;6:1-6.

3. Tragni E, Galli CL, Tubaro A, Del Negro P, Della LR. Anti-inflammatory activity of Echinacea angustifolia fractions separated on the basis of molecular weight. Pharmacol Res Commun 1988;20(S5):87-90.
4. Ditteova G, Velebny S, Hrckova G. Modulation of liver fibrosis and pathological changes in mice infected with Mesocestoides corti (M. vogae) after administration of glucan and liposomized glucan in combination with vitamin C. J Helmintol 2003;77:219-26.

5. Verlhac V, Obach A, Gahaudan J, Schuep W, Hole R. Immunomodulation by dietary vitamin $C$ and glucan in rainbow trout (Oncorhynchus mykiss). Fish Shellfish Immunol 1998;6:409-24.

6. Vetvicka V, Baigorri R, Zamarreno AM, Garcia-Mina JM, Yvin JC. Glucan and humic acid: Synergistic effects on the immune system. J Med Food 2010;13:863-69.

7. Vetvicka V, Vancikova Z. Synergistic effects of glucan and resveratrol. Afr J Biochem Res 2010;4:105-10.

8. Vetvicka V, Volny T, Saraswat-Ohri S, Vashishta A, Vancikova Z, Vetvickova J. Glucan and resveratrol complex - possible synergistic effects on immune system. Biomed Pap Med Fac Univ Palacky Olomouc Czech Repub 2007,151(1):41-6.

9. Vetvicka V, Vetvickova J. Combination of glucan, resveratrol and vitamin C demonstrates strong anti-tumor potential. Anticancer Res 2012;32:82-8

10. Vetvicka V, Vetvickova J. Physiological effects of different types of $\beta$-glucan. Biomed Pap Med Fac Univ Palacky Olomouc Czech Repub 2007;151(2):225-231.

11. Vetvicka V, Vetvickova J. $\beta 1,3-G l u c a n:$ Silver bullet or hot air? Open Glycoscience 2010;3:1-6.

12. Vetvicka V, Fornusek L, Kopecek J, Kaminkova J, Kasparek L, Vranova M. Phagocytosis of human blood leukocytes: A simple micromethod. Immunol Lett 1982;5:97-100.

13. Vetvicka V, Holub M, Kovaru H, Siman P, Kovaru F. Alpha-fetoprotein and phagocytosis in athymic nude mice. Immunol Lett 1988;19:95-8.

14. Fernandez-Botran R, Vetvicka V. Advanced Methods in Cellular Immunology, CRC Press, Boca Raton, 2000.

15. Green SJ, Nacy CA. Antimicrobial and immunopathological effects of cytokine-induced nitric oxide synthesis. Curr Opin Infect Dis 1993;6:284-396.

16. Vetvicka V, Yvin J-C. Effects of marine beta-1,3 glucan on immune reaction. Int Immunopharmacol 2004;4,721-30.

17. Vetvicka V, Vetvickova J. An evaluation of the immunological activities of commercially available $\beta 1,3$-glucans. J American Nutr Assoc 2007; 10:25-31.

18. Lawrence HS, Valentin FT. Transfer Factor and other mediators of cellular immunity. Am J Pathol 1970;60:437-47.

19. Sanchez-Gonzales DJ, Sosa-Luna CA, Vasquez-Moctezuma O. Transfer factors in medical therapy. Med Clin 2011;137:273-77.

20. Ghoneum M, Agrawal S. Activation of human monocyte-derived dendritic cells in vitro by the biological response modifier arabinoxylan rice bran (MGN-3/Biobran). Int J Immunopathol Pharmacol 2011;34:941-8

21. Ghoneum M, Gollapudi S. Synergistic apoptotic effect of arabinoxylan rice bran (MGN-3/Biobran) and curcumin (turmeric) on human multiple myeloma cell line U266 in vitro. Neoplasma 2011;58:118-23.

22. Nerren JR, Kogut MH. The selective Dectin-1 agonist, curdlan, induces an oxidative burst response in chicken heterophils and peripheral blood mononuclear cells. Vet Immunol Immunopathol 2009;127:207-22.

23. Vetvicka V, Dvorak B, Vetvickova J, Richter J, Krizan J, Sima P, Yvin J-C. Orally-administered marine (1-->3)-beta-D-glucan stimulates both humoral and cellular immunity. Int J Biol Macromol 2007;40:291-8.

24. Huang H, Ostroff GR, Lee CK, Specht CA, Levitz SM. Robust stimulation of humoral and cellular immune responses following vacination with antigen-loaded beta-glucan particles. mBio. 2010;1(3):e0016410. doi:10.1128/mBio.00164-10. 